\title{
Impact of Left Ventricular Ejection Fraction on Recurrent Ventricular Tachyarrhythmias in Recipients of Implantable Cardioverter Defibrillators
}

Jonas Rusnak $^{\mathrm{a}}$ Michael Behnes $^{\mathrm{a}}$ Christel Weiß $^{\mathrm{b}}$ Christoph Nienaber $^{\mathrm{c}}$ Linda Reiser $^{\mathrm{a}}$ Tobias Schupp ${ }^{\mathrm{a}}$ Armin Bollow $^{\mathrm{a}}$ Gabriel Taton ${ }^{\mathrm{a}}$ Thomas Reichelt $^{\mathrm{a}}$ Dominik Ellguth ${ }^{\mathrm{a}}$ Niko Engelke ${ }^{\mathrm{a}}$ Kathrin Weidner ${ }^{\mathrm{a}}$

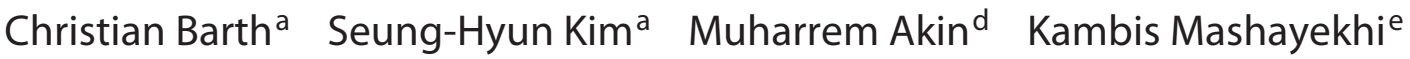
Dirk Große Meininghaus ${ }^{f}$ Martin Borggrefe ${ }^{a}$ Ibrahim Akin ${ }^{a}$

\footnotetext{
${ }^{a}$ First Department of Medicine, University Medical Centre Mannheim (UMM), Faculty of Medicine Mannheim, University of Heidelberg, European Center for AngioScience (ECAS), and DZHK (German Center for Cardiovascular Research) partner site Heidelberg/Mannheim, Mannheim, Germany; ${ }^{b}$ Institute of Biomathematics and Medical Statistics, University Medical Center Mannheim (UMM), Faculty of Medicine Mannheim, University of Heidelberg, Mannheim, Germany; ${ }^{\mathrm{C}}$ Royal Brompton and Harefield Hospitals, NHS, London, UK; ${ }^{\mathrm{d}}$ Department of Cardiology and Angiology, Hannover Medical School, Hannover, Germany; ${ }^{e}$ Clinic for Cardiology and Angiology II, Universitaetszentrum Freiburg Bad Krozingen, University of Freiburg, Bad Krozingen, Germany; ${ }^{\mathrm{f} D e p a r t m e n t}$ of Cardiology, Carl-Thiem-Klinikum Cottbus, Cottbus, Germany
}

\section{Keywords}

Ventricular tachycardia - Ventricular fibrillation · Mortality · Left ventricular ejection fraction $\cdot$ Heart failure $\cdot$ Implantable cardioverter defibrillator

\footnotetext{
Abstract

Objective: This study evaluates the impact of left ventricular ejection fraction (LVEF) on recurrences of ventricular tachyarrhythmias in recipients of implantable cardioverter defibrillator (ICD). Background: Data regarding recurrences of ventricular tachyarrhythmias in ICD recipients according to LVEF is limited. Methods: A large retrospective registry was used, including all consecutive ICD recipients with episodes of ventricular tachycardia (VT) or fibrillation (VF) from 2002 to 2016. Patients with LVEF $<35 \%$ were compared to patients with LVEF $\geq 35 \%$. The primary end point was first recurrences
}

$\begin{aligned} & \text { karger@karger.com } \\ & \text { www.karger.com/crd }\end{aligned}$
Karger $\%$

of ventricular tachyarrhythmias at 5 years. Secondary end points were ICD-related therapies, rehospitalization, and allcause mortality at 5 years. Cox regression, Kaplan Meier, and propensity score matching analyses were applied. Results: $\mathrm{A}$ total of 528 consecutive ICD recipients were included (51\% with LVEF $\geq 35 \%$ and $49 \%$ with LVEF <35\%). LVEF <35\% was associated with reduced freedom from recurrent ventricular tachyarrhythmias (40 vs. $49 \%$, log rank $p=0.014$; hazard ratio $[\mathrm{HR}]=1.381 ; 95 \%$ confidence interval $[\mathrm{Cl}] 1.066-1.788 ; p=$ $0.034)$, mainly attributed to recurrent sustained VT in primary preventive ICD recipients. Accordingly, LVEF $<35 \%$ was associated with reduced freedom from first appropriate ICD therapies (28 vs. $41 \%$, log rank $p=0.001 ; \mathrm{HR}=1.810 ; 95 \% \mathrm{Cl}$ 1.185-2.766; $p=0.001)$. Finally, LVEF $<35 \%$ was associated

J.R. and M.B. contributed equally to this work. 
with a higher rate of rehospitalization ( 23 vs. $34 \% ; p=0.005$ ) and all-cause mortality at 5 years ( 13 vs. $29 \% ; p=0.001)$. Conclusion: LVEF $<35 \%$ was associated with reduced freedom from recurrent ventricular tachyarrhythmias, appropriate device therapies, rehospitalization and all-cause mortality secondary to index ventricular tachyarrhythmias.

(c) 2020 S. Karger AG, Basel

\section{Introduction}

Sudden cardiac death (SCD) following ventricular tachyarrhythmias is a major threat in patients suffering from heart failure [1-3]. Ventricular tachyarrhythmias are common in patients with ischemic and non-ischemic cardiomyopathy (ICMP and NICMP) [4-9]. The implantable cardioverter defibrillator (ICD) is recommended to reduce SCD in patients with New York Heart Association (NYHA) class II or III, left ventricular ejection fraction (LVEF) $<35 \%$, and optimal medical heart failure therapy of at least 3 months [1].

Regarding secondary prevention, the ICD is recommended for patients with hemodynamically unstable ventricular tachycardia (VT) or fibrillation (VF) in the absence of reversible causes or developing $48 \mathrm{~h}$ after an acute myocardial infarction (AMI) [1]. There are three major randomized controlled trials (RCT), AVID, CIDS, and $\mathrm{CASH}$, which evaluated the prognostic impact of an ICD in cardiac arrest survivors with documented ventricular tachyarrhythmias and reduced LVEF [10-13]. The average LVEF recorded in these trials was 32\% (AVID), $34 \%$ (CIDS), and $46 \%$ (CASH), respectively, and the meta-analysis reported no benefit of ICD supply in patients with LVEF $\geq 35 \%$ [10].

Regarding the indication of an ICD for primary prevention, several RCT evaluated patients with LVEF $<35 \%$ (range 21-30\%) due to both ischemic and non-ischemic causes [14-21]. It was demonstrated that patients with LVEF $<35 \%$ benefited from primary preventive ICD implantation with an adjusted relative risk reduction ranging from -23 to $-51 \%$ [14-21]. A meta-analysis of data from the SCD-HeFT and the MADIT-II trials, which evaluated patients indicated for a primary preventive ICD due to $L V E F<35 \%$, reported that the decrease of LVEF of at least one percentage was associated with an increasing risk of appropriate ICD shocks [22].

However, it is currently debated whether the decision for ICD implantation should be made based solely on decreased LVEF values, as recommended by international guidelines [1,23]. Notably, at least $40 \%$ of SCD occur in patients with LVEF $>40 \%$, whereas only $13 \%$ of SCD can be attributed to patients with LVEF $<40 \%$. Furthermore, $45 \%$ of SCD occur in patients without any medical history related to cardiac diseases while $2 \%$ of SCD are related to genetic disorders [24].

Therefore, this study evaluates the impact of LVEF on secondary prognostic outcomes in ICD recipients with index episodes of ventricular tachyarrhythmias, focusing on recurrences of ventricular tachyarrhythmias, ICD-related therapies, rehospitalization, and all-cause mortality.

\section{Methods}

Data Collection and Documentation

All consecutive ICD recipients presenting with ventricular tachyarrhythmias on admission to our institution in 2002-2016 were included. All relevant clinical data related to the index event, the recurrences of ventricular tachyarrhythmia, and rehospitalizations (as documented during routine clinical care by the treating physicians and medical staff, including independent cardiologists), was retrospectively derived from the electronic hospital information system, daily documentation in patient files, discharge letters, diagnostic test results, and the laboratory system. Data were transferred into a standardized electronic database, where its quality and accuracy were re-assessed by two independent cardiologists (M.B. and I.A.).

Ventricular tachyarrhythmias consisted of VT and VF, as defined by current international guidelines $[1,4]$. Sustained VT was defined by a duration of $>30 \mathrm{~s}$ or causing hemodynamic collapse within $30 \mathrm{~s}$ and non-sustained VT by a duration of $<30 \mathrm{~s}$, both with wide QRS complex ( $\geq 120 \mathrm{~ms}$ ) at a rate of $>100 \mathrm{bpm}$ [1]. Ventricular tachyarrhythmias at index were documented by 12-lead electrocardiogram (ECG), ECG tele-monitoring, ICD, or in the case of an unstable course or during resuscitation by external defibrillator monitoring. Documented VF was treated by ICD-related shock or external defibrillation and in the case of prolonged instability with additional intravenous anti-arrhythmic drugs during cardiopulmonary resuscitation (CPR). Electrical storm (ES) was defined as $\geq 3$ episodes of ventricular tachyarrhythmias requiring appropriate device therapies and occurring during $24 \mathrm{~h}[1,25]$.

Clinical data comprised baseline characteristics, prior medical history, prior medical treatment, length of index stay, detailed findings of laboratory values at baseline, and data derived from all non-invasive or invasive cardiac diagnostics and device therapies, such as coronary angiography, electrophysiological examination, 12-lead or Holter ECG, echocardiography, cardiac magnetic resonance imaging (cMRI), pharmacological therapies, and the ICD protocol.

The following cardiac devices were allowed: ICD, cardiac resynchronization therapy with defibrillator (CRT-D), and subcutaneous ICD (s-ICD). ICD recipients presented routinely every 3-6 months to check the device, and unscheduled in the case of noticed device interrogations at our clinic. The device was set and programmed during routine clinical care according to current international guidelines compiled by cardiologists specialized in electrophysiology $[1,4,26]$. Device recordings were re-evaluated ret- 
rospectively by independent cardiologists who were blinded to the final data analysis.

Every rehospitalization, either ambulatory or in-hospital at our institution, was reviewed and documented for recurrent ventricular tachyarrhythmias, in-hospital death, and upcoming relevant cardiac events.

This study is derived from an analysis of the "Registry of Ma-

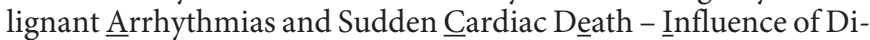
agnostics and Interventions (RACE-IT)," a single-centre registry including consecutive patients presenting with ventricular tachyarrhythmias and aborted cardiac arrest who were admitted to the University Medical Center Mannheim (UMM), Germany (ClinicalTrials.gov ID: NCT02982473) in 2002-2016.

\section{Inclusion and Exclusion Criteria}

Only patients with an activated ICD were included. All patients had a documented episode of ventricular tachyarrhythmias; this defines the index event. Each patient was counted only once for inclusion when presenting with the first episode of ventricular tachyarrhythmias. All analyzed patients had to survive index hospitalization.

For the analysis, risk-stratification was performed according to the presence of LVEF $<35 \%$ or $\geq 35 \%$ according to European guidelines [27]. LVEF values were retrieved from standardized transthoracic echocardiographic examinations, usually performed before hospital discharge of survivors, to assess realistic LVEF values. In minor part, and only if available, earlier LVEF values assessed on admission or during a stay in ICU were used.

Patients without complete follow-up data regarding mortality were excluded from the study. Each patient was counted only once for inclusion when presenting with the first episode of ventricular tachyarrhythmias.

\section{Primary and Secondary End Points}

Follow-up was set at 5 years for all end points. The primary end point was the first recurrence of ventricular tachyarrhythmias (VT or VF) as documented in the ICD protocol. Secondary end points were overall recurrences at follow-up, recurrences per patient, associated appropriate or inappropriate device therapies (first, overall, and per patient), first rehospitalization, and all-cause mortality at follow-up. Further stratification into subgroups of primary or secondary prevention and appropriate or inappropriate device therapies was performed.

Appropriate device therapy was defined as device interrogation in the presence of VT or VF including anti-tachycardia pacing (ATP), ICD shock, or both. Inappropriate device therapy was defined as ATP or ICD shock in the absence of VT or VF. First rehospitalization comprised rehospitalization due to VT, VF, AMI, acute $\mathrm{HF}$, and inappropriate device therapy.

All-cause mortality was documented using our electronic hospital information system and by directly contacting state resident registration offices (The Bureau of Mortality Statistics) all across Germany. Identification of patients was verified by means of their name, surname, date of birth, and residential address.

\section{Statistical Methods}

Quantitative data is presented as mean \pm standard error of the mean (SEM) or median and inter-quartile range (IQR), depending on the distribution of the data. The Student $t$ test was used to compare normally distributed data and the Mann-Whitney U test for non-parametric data. Deviations from a Gaussian distribution were tested by the Kolmogorov-Smirnov test. Spearman's rank correlation for non-parametric data was used to test univariate correlations. Qualitative data are presented as absolute and relative frequencies and compared using the $\chi^{2}$ test or Fisher exact test, as appropriate.

Firstly, the univariate Kaplan-Meier method was applied to evaluate differences in primary and secondary end points within the entire unmatched cohort between patients with LVEF $<35 \%$ and $\geq 35 \%$. Furthermore, differences were tested in subgroups of primary versus secondary prevention. Hazard ratios (HRs) are given together with $95 \%$ confidence intervals (CIs). Secondly, multivariable Cox regression models were developed using the "forward selection" option, where only statistically significant $(p<0.05)$ or clinically relevant variables were included and analyzed simultaneously. Predefined variables used for multi-variable Cox regressions included: age, gender, diabetes mellitus, chronic kidney disease, atrial fibrillation $(\mathrm{AF}), \mathrm{CPR}$, coronary artery disease (CAD), administration of angiotensin-converting enzyme (ACE) inhibitors, $\beta$ blocker, amiodarone, and LVEF $<35 \%$. Patients without complete follow-up were censored (accepted lost to follow-up rate $<10 \%)$.

The result of a statistical test was considered significant for $p<$ 0.05 , a statistical trend was defined as $p<0.10$. SAS v9.4 (SAS Institute Inc., Cary, NC, USA) and SPSS v25 (IBM, Armonk, NY, USA) were used for statistics.

\section{Propensity Score Matching}

In an RCT, patients with or without a specific treatment would have a $50 \%$ chance of being treated, and balanced measured and unmeasured baseline characteristics would be expected. In an observational study on real-life patients, the specific groups would not be randomized, resulting in the chances of receiving treatment varying from 0 to $100 \%$, including imbalances in baseline characteristics. Consecutively, differences in outcomes in the specific groups might therefore also be explained by the heterogenous distribution of the baseline characteristics. To reduce this selection bias, we used 1:1 propensity scores for LVEF $<35$ and $\geq 35 \%$ to assemble a matched cohort in which patients would be well-balanced on predefined baseline characteristics. 1:1 propensity score matching was performed including the entire study cohort performing a non-parsimonious multivariable logistic regression model using patients with $\mathrm{LVEF}<35 \%$ as the dependent variable $[28,29]$. Propensity scores were created according to the following independent variables: age, gender, diabetes mellitus, and chronic kidney disease (glomerular filtration rate $<60 \mathrm{~mL} / \mathrm{min} / 1.73 \mathrm{~m}^{2}$ ). Based on the propensity score values counted by logistic regression, for each patient in the LVEF $<35 \%$ group, one patient in the LVEF $\geq 35 \%$ group with a similar propensity score value was found (accepted difference in propensity score value $<5 \%$ ).

\section{Results}

\section{Study Population}

A total of 528 consecutive ICD recipients, 51\% with LVEF $\geq 35 \%$ and $49 \%$ with LVEF $<35 \%$, who survived an index episode of ventricular tachyarrhythmias were in- 
Table 1. Patient characteristics and comorbidities before and after propensity score matching according to left ventricular function

\begin{tabular}{|c|c|c|c|c|c|c|}
\hline \multirow[t]{2}{*}{ Characteristic } & \multicolumn{3}{|c|}{ Before matching $(n=528)$} & \multicolumn{3}{|c|}{ After matching $(n=432)$} \\
\hline & $\begin{array}{l}\text { LVEF } \geq 35 \% \\
(n=271 ; 51 \%)\end{array}$ & $\begin{array}{l}\text { LVEF <35\% } \\
(n=257 ; 49 \%)\end{array}$ & $p$ value & $\begin{array}{l}\text { LVEF } \geq 35 \% \\
(n=216 ; 50 \%)\end{array}$ & $\begin{array}{l}\text { LVEF }<35 \% \\
(n=216 ; 50 \%)\end{array}$ & $p$ value \\
\hline Median age, years (range) & $55(21-75)$ & $62(44-75)$ & 0.001 & $67(21-80)$ & $67(42-87)$ & 1.000 \\
\hline Male gender & $216(79)$ & $210(82)$ & 0.504 & $176(82)$ & $176(81)$ & 0.072 \\
\hline \multicolumn{7}{|l|}{ Ventricular tachyarrhythmias } \\
\hline Ventricular tachycardia & $166(61)$ & $203(79)$ & 0.001 & $169(74)$ & $164(76)$ & 0.657 \\
\hline Ventricular fibrillation & $105(39)$ & $054(21)$ & & $56(26)$ & $52(24)$ & \\
\hline \multicolumn{7}{|l|}{ Cardiovascular risk factors } \\
\hline Arterial hypertension & $164(60)$ & $170(66)$ & 0.163 & $141(65)$ & $144(67)$ & 0.761 \\
\hline Diabetes mellitus & $69(25)$ & $69(27)$ & 0.698 & $60(28)$ & $59(27)$ & 0.914 \\
\hline Hyperlipidemia & $97(36)$ & $122(48)$ & 0.006 & $88(41)$ & $102(47)$ & 0.171 \\
\hline Smoking & $70(26)$ & $79(31)$ & 0.201 & $61(28)$ & $69(31)$ & 0.401 \\
\hline Cardiac family history & $40(15)$ & $35(14)$ & 0.720 & $33(15)$ & $32(15)$ & 0.893 \\
\hline \multicolumn{7}{|l|}{ Comorbidities } \\
\hline Chronic kidney disease & $98(37)$ & $128(50)$ & 0.002 & $91(42)$ & $94(44)$ & 0.771 \\
\hline Atrial fibrillation & $75(28)$ & $98(38)$ & 0.010 & $64(30)$ & $83(38)$ & 0.054 \\
\hline Acute myocardial infarction & $39(14)$ & $31(12)$ & 0.440 & $26(12)$ & $28(13)$ & 0.771 \\
\hline Cardiogenic shock & $27(10)$ & $20(8)$ & 0.386 & $17(8)$ & $19(9)$ & 0.728 \\
\hline Cardiomyopathy & $39(14)$ & $57(22)$ & 0.019 & $29(13)$ & $47(22)$ & 0.023 \\
\hline $\mathrm{CPR}$ & $59(22)$ & $21(8)$ & 0.001 & $31(14)$ & $21(10)$ & 0.323 \\
\hline \multicolumn{7}{|l|}{ Medication at discharge } \\
\hline$\beta$ Blocker & $225(83)$ & $244(95)$ & 0.001 & $182(84)$ & $204(94)$ & 0.001 \\
\hline ACE inhibitor/ARB & $180(66)$ & $194(76)$ & 0.019 & $146(68)$ & $163(76)$ & 0.070 \\
\hline Aldosterone antagonist & $24(9)$ & $61(24)$ & 0.001 & $18(8)$ & $47(22)$ & 0.001 \\
\hline Amiodarone & $34(13)$ & $67(26)$ & 0.001 & $26(12)$ & $62(29)$ & 0.001 \\
\hline \multicolumn{7}{|l|}{ ECG interval (mean \pm SEM) } \\
\hline PQ & $175 \pm 5$ & $179 \pm 6$ & 0.590 & $173 \pm 5$ & $178 \pm 7$ & 0.559 \\
\hline QRS & $97 \pm 3$ & $124 \pm 5$ & 0.001 & $99 \pm 4$ & $122 \pm 6$ & 0.001 \\
\hline QT & $417 \pm 7$ & $413 \pm 5$ & 0.724 & $419 \pm 8$ & $416 \pm 5$ & 0.743 \\
\hline
\end{tabular}

Values express n (\%), unless otherwise indicated. Bold type denotes significance. LVEF, left ventricular ejection fraction; CPR, cardiopulmonary resuscitation; ACE, angiotensin-converting-enzyme; ARB, angiotensin receptor blocker.

cluded. Baseline characteristics are given in Table 1. Most patients were males (79 and 82\%).

Patients with LVEF $<35 \%$ were older than those with LVEF $\geq 35 \%$ (62 vs. 55 years, $p=0.001$ ). In general, VT was more common than VF $(61-79 \%$ vs. $21-39 \%, p=$ 0.001). Patients with LVEF $<35 \%$ had a higher rate of VT, atrial fibrilation, chronic kidney disease, hyperlipidaemia, and cardiomyopathy, and were more often treated with $\beta$ blockers, angiotensin-converting enzyme (ACE) inhibitors/angiotensin receptor blockers (ARB), aldosterone antagonists, and amiodarone. Furthermore, patients with LVEF $<35 \%$ showed prolonged QRS duration. No further significant differences were observed between groups.

After propensity score matching, both groups had comparable rates of baseline characteristics, despite dif- fering rates of cardiomyopathy, medication at discharge, and QRS duration which were not included in the matching process (Table 1 ).

Table 2 outlines the ICD-related data of the study population. Most patients had an activated transvenous ICD (96 vs. $87 \%$ ), whereas transvenous CRT-D (3 vs. $11 \%$ ) or s-ICD ( 1 vs. $2 \%$ ) were present in minor part. Regarding the type of ICD, there were significant differences between both groups, in the general cohort, as well as in the propensity score-matched cohort. The indication for ICD implantation was equally distributed for primary and secondary prevention. The median detection thresholds for VT (172 vs. $174 \mathrm{bpm}$ ) and for VF (217 bpm) were comparable in both groups, as well as the median cycle length of VT (range 315-323 ms). 
Table 2. ICD and follow-up data before and after propensity score matching according to left ventricular function

\begin{tabular}{|c|c|c|c|c|c|c|}
\hline & \multicolumn{3}{|c|}{ Before matching $(n=528)$} & \multicolumn{3}{|c|}{ After matching $(n=432)$} \\
\hline \multicolumn{7}{|l|}{ Characteristic } \\
\hline \multicolumn{7}{|l|}{ Type of ICD } \\
\hline ICD & 259 (96) & $225(87)$ & 0.003 & 207 (96) & $188(87)$ & 0.005 \\
\hline \multicolumn{7}{|l|}{ Implant indication } \\
\hline Primary prevention & $109(40)$ & $121(47)$ & 0.124 & $98(45)$ & $102(47)$ & 0.700 \\
\hline Secondary prevention & $162(60)$ & $136(53)$ & & $118(55)$ & $114(53)$ & \\
\hline \multicolumn{7}{|l|}{ ICD programming } \\
\hline VT detection threshold ${ }^{\mathrm{a}}$, bpm & $172(167-76)$ & $174(167-76)$ & 0.075 & $167(161-71)$ & $165(165-71)$ & 0.654 \\
\hline VF detection threshold ${ }^{\mathrm{a}}$, bpm & $217(214-24)$ & $217(214-14)$ & 0.920 & $218(214-21)$ & $216(214-16)$ & 0.944 \\
\hline VT cycle length ${ }^{\mathrm{a}}, \mathrm{ms}$ & $315(280-40)$ & $323(288-50)$ & 0.250 & $312(280-40)$ & $325(295-50)$ & 0.238 \\
\hline \multicolumn{7}{|l|}{ Primary end point } \\
\hline Sustained VT & $54(20)$ & $85(33)$ & 0.001 & $49(23)$ & $72(33)$ & 0.015 \\
\hline VF & $19(7)$ & $14(5)$ & 0.465 & $16(7)$ & $11(5)$ & 0.314 \\
\hline Electrical storm & $13(5)$ & $21(8)$ & 0.112 & $11(5)$ & $18(8)$ & 0.178 \\
\hline \multicolumn{7}{|l|}{ Secondary end points } \\
\hline \multicolumn{7}{|l|}{ Overall recurrences at follow-up } \\
\hline Non-sustained VT & $49(18)$ & $51(20)$ & 0.591 & $36(17)$ & $38(18)$ & 0.798 \\
\hline Sustained VT & $67(25)$ & $102(40)$ & 0.001 & $59(27)$ & $85(39)$ & 0.008 \\
\hline $\mathrm{VF}$ & $24(9)$ & $29(11)$ & 0.346 & $21(10)$ & $22(10)$ & 0.872 \\
\hline Electrical storm & $13(5)$ & $21(8)$ & 0.112 & $11(5)$ & $18(8)$ & 0.178 \\
\hline \multicolumn{7}{|c|}{ Recurrences per patient (mean \pm SEM) } \\
\hline Non-sustained VT & $2.4 \pm 0.74$ & $8.1 \pm 3.2$ & 0.076 & $2.7 \pm 0.9$ & $8.0 \pm 3.7$ & 0.172 \\
\hline Sustained VT & $4.0 \pm 0.97$ & $6.5 \pm 1.8$ & 0.211 & $4.3 \pm 1.0$ & $6.4 \pm 2.0$ & 0.347 \\
\hline Appropriate ATP only & $57(21)$ & $85(33)$ & 0.002 & $53(25)$ & $71(33)$ & 0.059 \\
\hline Inappropriate device therapy & $32(12)$ & $37(14)$ & 0.378 & $26(12)$ & $30(14)$ & 0.579 \\
\hline \multicolumn{7}{|c|}{ Device therapies per patient (mean \pm SEM) } \\
\hline Appropriate shock & $0.5 \pm 0.2$ & $0.9 \pm 0.2$ & 0.128 & $0.4 \pm 0.2$ & $0.9 \pm 0.2$ & 0.098 \\
\hline Appropriate ATP only & $3.4 \pm 0.8$ & $5.3 \pm 1.7$ & 0.317 & $4.0 \pm 1.0$ & $5.8 \pm 2.0$ & 0.424 \\
\hline Inappropriate shock & $0.6 \pm 0.2$ & $0.27 \pm 0.1$ & 0.172 & $0.7 \pm 0.3$ & $0.3 \pm 0.07$ & 0.161 \\
\hline Inappropriate ATP only & $0.1 \pm 0.02$ & $0.1 \pm 0.1$ & 0.529 & $0.08 \pm 0.04$ & $0.04 \pm 0.02$ & 0.300 \\
\hline \multicolumn{7}{|l|}{ Rehospitalization } \\
\hline Overall & $63(23)$ & $88(34)$ & 0.005 & $56(26)$ & $84(39)$ & 0.004 \\
\hline VT & $17(6)$ & $31(12)$ & 0.020 & $16(7)$ & $26(12)$ & 0.108 \\
\hline VF & $6(2)$ & $6(2)$ & 0.921 & $5(2)$ & $5(2)$ & 0.994 \\
\hline AMI & $3(1)$ & $4(2)$ & 0.648 & $3(1)$ & $4(2)$ & 0.708 \\
\hline Acute heart failure & $15(6)$ & $22(9)$ & 0.170 & $14(7)$ & $18(8)$ & 0.471 \\
\hline Inappropriate device therapy & $10(4)$ & $14(6)$ & 0.328 & $8(4)$ & $12(6)$ & 0.365 \\
\hline Other & $12(4)$ & $11(4)$ & 0.941 & $10(5)$ & $9(4)$ & 0.806 \\
\hline All-cause mortality, at 5 years & $36(13)$ & $74(29)$ & 0.001 & $34(16)$ & $58(27)$ & 0.005 \\
\hline
\end{tabular}

Values are expressed as $n$ (\%), unless otherwise indicated. Bold type denotes significance. ${ }^{\text {a }}$ Expressed as median (IQR). ATP, antitachycardia pacing; HF, heart failure; VF, ventricular fibrillation; VT, ventricular tachycardia; AMI, acute myocardial infarction; ICD, implantable cardioverter defibrillator; CRT-D, cardiac resynchronisation therapy and defibrillator; s-ICD, subcutaneous implantable cardioverter defibrillator. 


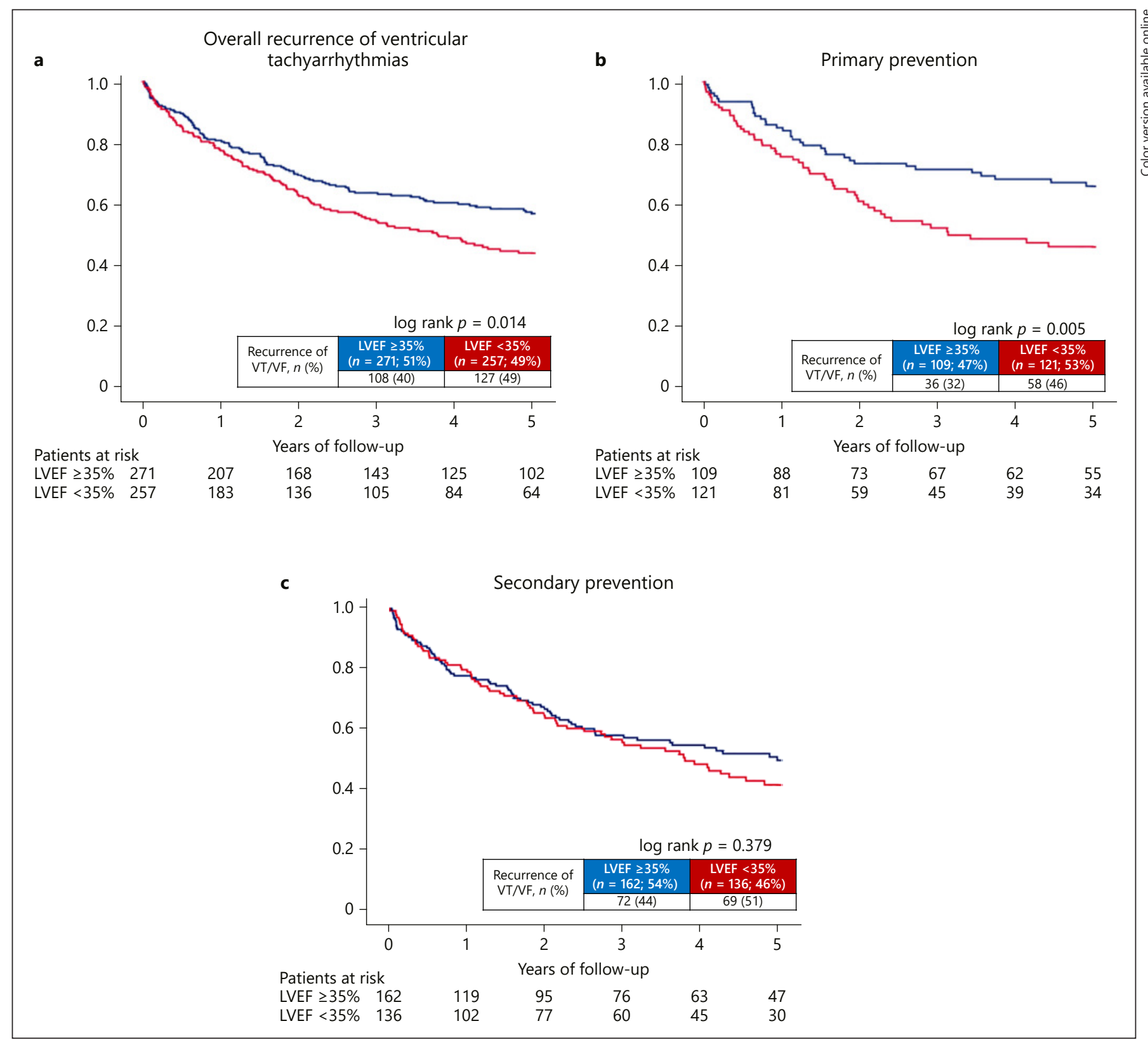

Fig. 1. Freedom from first recurrence of ventricular tachyarrhythmias (a), stratified to primary (b) and secondary preventive ICD recipients $(\mathbf{c})$.

\section{Follow-Up Data and Primary and Secondary End}

Points

At least $90 \%$ of patients were followed up regularly within a period of 5 years (1,825 days) with at least one ICD check-up every 6-12 months.

The primary end point of freedom from first episodes of recurrent ventricular tachyarrhythmias was decreased in patients with $\mathrm{LVEF}<35 \%$ compared to those with
LVEF $\geq 35 \%$ ( 40 vs. $49 \%$, log-rank $p=0.014 ; \mathrm{HR}=1.381$ 95\% CI 1.066-1.788; $p=0.034$ ) (Table 2; Fig. 1a). Notably, this difference was mainly observed in patients with a primary preventive ICD ( 32 vs. $46 \%$; $\log$ rank $p=0.005$; $\mathrm{HR}=1.810 ; 95 \%$ CI 1.185-2.766; $p=0.006)$, but not in secondary preventive ICD recipients $(\log \operatorname{rank} p=0.379)$ (Fig. 1b, c). Differences in recurrences of ventricular tachyarrhythmias can presumably be attributed to the 


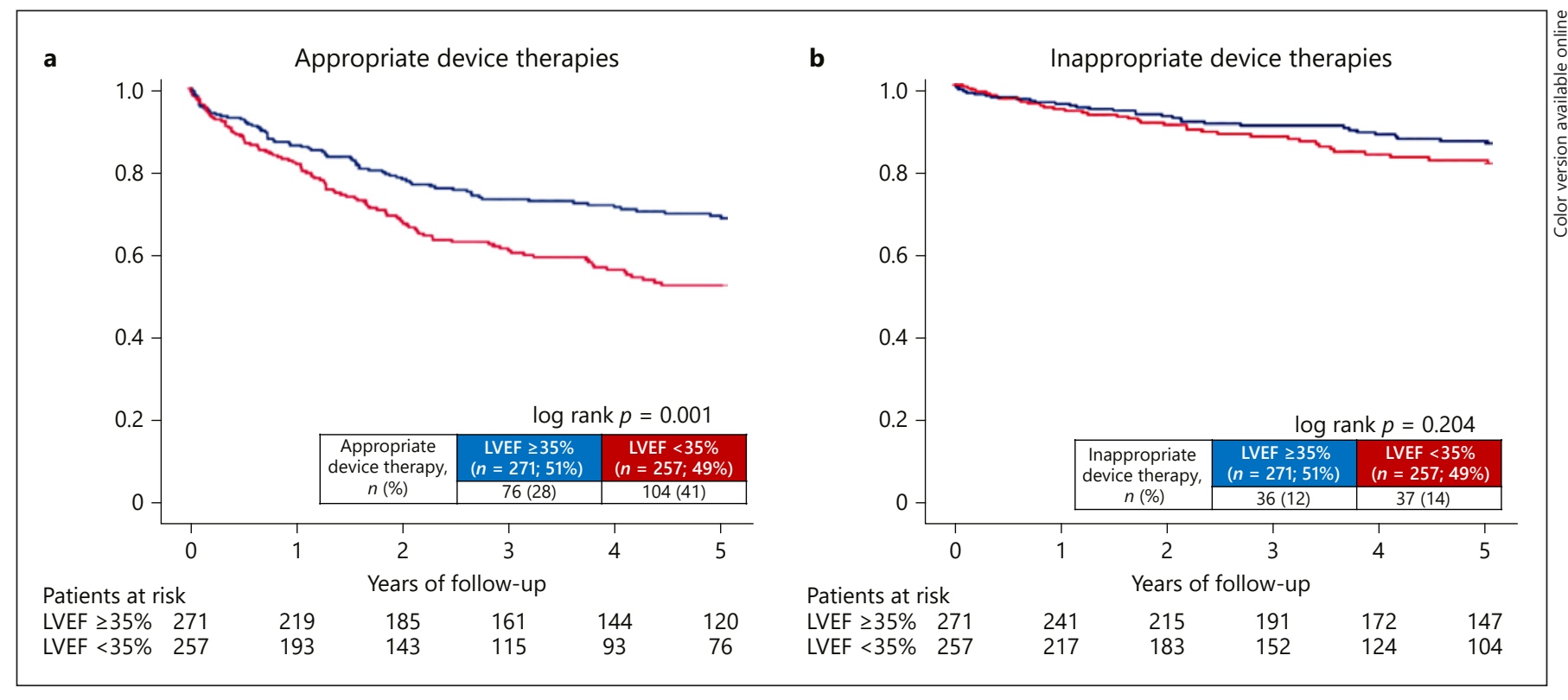

Fig. 2. LVEF $<35 \%$ decreased freedom from appropriate device therapies (a) while not affect inappropriate device therapies (b).

higher rate of sustained VT in LVEF $<35 \%$ patients $(20$ vs. $33 \%, p=0.001$ ), which is also reflected by an increasing rate of overall sustained VT ( 25 vs. $40 \%, p=0.001$ ) (Table 2).

Regarding secondary end points, freedom from overall first appropriate device therapies was decreased in LVEF $<35 \%$ at 5 years $(28$ vs. $41 \%, \log$ rank $p=0.001 ; \mathrm{HR}=$ 1.656; 95\% CI 1.231-2. 227; $p=0.001$ ) (Fig. 2a). This difference was presumably driven both by increasing rates of appropriate ATP (16 vs. $21 \%$ ) and appropriate shock (12 vs. 19\%) in LVEF <35\%. Inappropriate device therapies were comparable in both groups (Table 2).

Moreover, patients with LVEF $<35 \%$ revealed higher rates of overall first rehospitalization ( 23 vs. $34 \%$ ), mainly attributed to VT recurrence, and a higher rate of allcause mortality at 5 years (13 vs. 29\%) (Table 2).

Notably, the primary end point of freedom from first episodes of recurrent ventricular tachyarrhythmias was still reduced in $\mathrm{LVEF}<35 \%$ versus $\mathrm{LVEF} \geq 35 \%$ after propensity score matching ( 40 vs. $46 \%$; $\mathrm{HR}=1.316$; $95 \% \mathrm{CI}$ $0.985-1.759 ; p=0.063$ ) (Fig. 3a). This was still mainly attributed to sustained VT ( 23 vs. $33 \%$; HR $=1.498 ; 95 \%$ CI $0.956-2.346 p=0.015$ ) (Fig. 3b). Regarding secondary end points, freedom from overall first appropriate device therapies was decreased in the LVEF $<35 \%$ group at 5 years after propensity score matching (30 vs. $38 \%$, log rank $p=0.024 ; \mathrm{HR}=1.441 ; 95 \%$ CI $1.048-1.982 ; p=$
0.001) (Fig. 3c). Moreover, after propensity score matching patients with LVEF $<35 \%$ revealed higher rates of overall first rehospitalization and all-cause mortality at 5 years (Table 2).

\section{Multivariable Cox Regression Models}

Even after multivariable adjustment, LVEF $<35 \%$ was still associated with a 1.3-fold higher risk of a first recurrence of ventricular tachyarrhythmias ( $\mathrm{HR}=1.296$; 95\% CI $0.982-1.712 ; p=0.067$ ) (Fig. 4, left panel). Accordingly, LVEF $<35 \%$ was still associated with a 1.5 -fold higher risk of appropriate device therapies $(\mathrm{HR}=1.459$; 95\% CI 1.058-2.011; $p=0.021$ ) (Fig. 4, right panel).

\section{Discussion}

This study evaluates the prognostic impact of LVEF on the primary end point of recurrences of ventricular tachyarrhythmias as well as on the secondary end points, consisting of device-related therapies, rehospitalization, and all-cause mortality at 5 years, in ICD recipients surviving episodes of ventricular tachyarrhythmias at index.

The data suggests that LVEF $<35 \%$ is associated with reduced freedom from first recurrent ventricular tachyarrhythmias and from first appropriate device-related therapies. LVEF $<35 \%$ may especially increase the rates of re- 


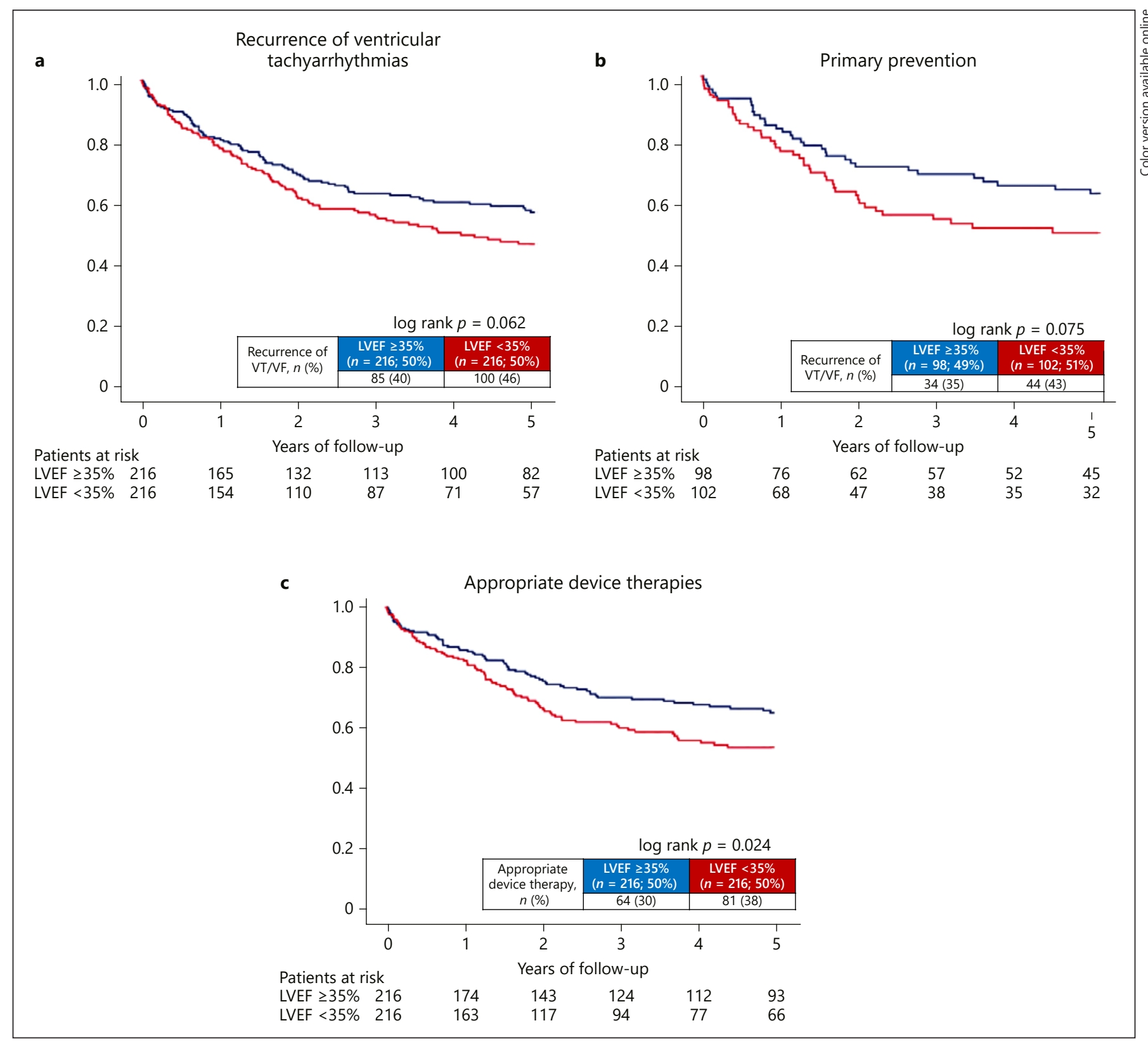

Fig. 3. Freedom from first recurrence of ventricular tachyarrhythmias (a), stratified to primary preventive ICD recipients (b), as well as freedom from first appropriate device therapies (c) in the matched cohort.

current sustained VT, whereas inappropriate device-related therapies were not affected. The adverse prognostic impact of $\mathrm{LVEF}<35 \%$ was also seen in a propensity scorematched cohort, revealing a trend towards a decrease in freedom from first recurrent ventricular tachyarrhythmias and a significant reduction regarding the freedom from first appropriate device-related therapies. The results showed consistency even after multi-variable adjust- ment. Furthermore, patients with LVEF $<35 \%$ revealed a higher rate of rehospitalization and all-cause mortality at 5 years, in both the unmatched and matched cohorts.

Regarding risk stratification for SCD, LVEF is the recommended risk marker in current international guidelines for decision-making for or against ICD implantation, especially for primary prevention [1]. A retrospective analysis of 349 patients with NICMP and ICD for 


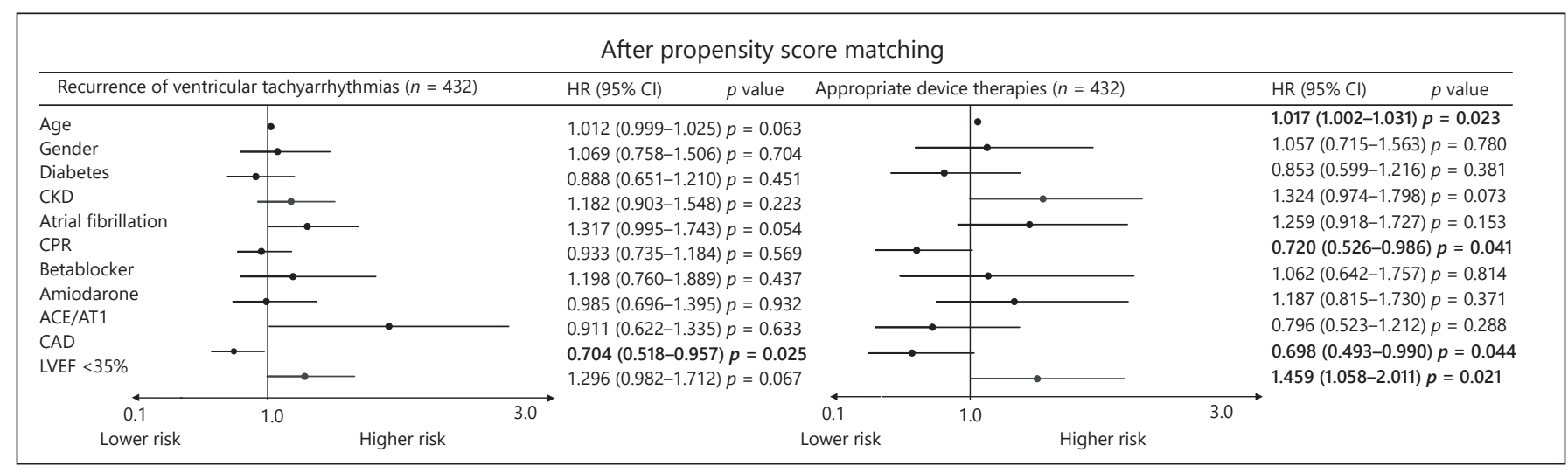

Fig. 4. $\mathrm{LVEF}<35 \%$ was still associated with an increased risk for recurrent ventricular tachyarrhythmias (left) and appropriate ICD therapies (right) after multivariable adjustment in Cox regression models. Bold type denotes significance.

primary $(70 \%)$ or secondary prevention $(30 \%)$ showed a lower risk of appropriate ICD shocks in patients with an improved LVEF of $\geq 35 \%$ [30]. These results were confirmed by a prospective cohort study performed by Zhang et al. [31], which included 538 ICMP and NICMP patients with LVEF $<30 \%$ and ICD or CRT-D for primary prevention. It was demonstrated that LVEF $<35 \%$ was associated with increased rates of all-cause mortality and appropriate shocks for ventricular tachyarrhythmias compared to patients with LVEF $\geq 35 \%$ at 4.9 years of follow-up. These findings are in line with this study, which demonstrates significant increases in recurrent sustained VT and appropriate device therapies in patients with $\mathrm{LVEF}<35 \%$ compared to patients with $\mathrm{LVEF} \geq 35 \%$. Therefore, LVEF in patients with a primary preventive ICD might be an appropriate risk predictor for recurrent ventricular tachyarrhythmias.

Risk prediction for secondary prevention is not based solely on LVEF. Therefore, LVEF plays a minor part in the evaluation of ICD therapy in patients with a history of ventricular tachyarrhythmias [1]. This approach was also confirmed in this study. Here, patients with a secondary preventive ICD revealed similar rates of recurrences of ventricular tachyarrhythmias irrespective of the underlying LVEF. In a sub-analysis of the CASH trial, which included patients with an aborted cardiac arrest due to sustained VT and consecutive secondary preventive ICD implantation, the degree of LVEF was evaluated as a risk predictor of death from any cause [11]. No significant differences between the patients with LVEF $<35$ and $\geq 35 \%$ were observed. In the AVID trial, including resuscitated patients due to sustained VT or VF, the benefit of ICD implantation due to secondary prevention was proven, irrespective of whether the LVEF was $<35$ or $\geq 35 \%$ [12]. These results indicate that implantation of an ICD for secondary prevention should not be based solely on LVEF reduction, which is supported by these findings.

Nevertheless, LVEF remains a reliable predictor of SCD in patients with structural heart disease, irrespective of the underlying aetiology [32]. However, there are still relevant limitations regarding the adequate identification of patients at risk. Accordingly, in this study almost half of the patients with LVEF $<35 \%$ showed no recurrence of ventricular tachyarrhythmias at 5 years. A meta-analysis of the SCD-HeFT and MADIT-II trials revealed that $80 \%$ of patients with LVEF $<35 \%$ and primary preventive ICD did not experience any appropriate ICD shock at 2.5 years [22]. A prospective observational trial including patients with primary and secondary preventive ICD revealed that LVEF was a poor predictor of appropriate ICD shocks, which is in clear contrast to the results of this study [33]. These controversial findings might be explained by the existence of pro-arrhythmic substrates, such as cardiac fibrosis or myocardial scarring, which are independent from LVEF or improvement of LVEF [31, 34]. The implantation of an ICD based only on LVEF assessment does not seem to be the best solution, due to the low sensitivity and specificity and the knowledge that SCD occurs also in patients with LVEF $>40 \%[23,24]$. Therefore, further studies are needed to evaluate those patients with LVEF $<35 \%$ without any risk and those patients with $\mathrm{LVEF} \geq 35 \%$ at high risk for SCD.

In conclusion, the study demonstrates that LVEF $<35 \%$ is associated with reduced freedom from recurrent 
ventricular tachyarrhythmias in ICD recipients who have already survived an episode of ventricular tachyarrhythmias, alongside with decreased freedom from appropriate device therapies, increasing rehospitalization, and allcause mortality at 5 years. Therefore, LVEF might improve risk prediction of the recurrence of ventricular tachyarrhythmias, especially in the setting of primary prevention, whereas further more precise risk markers are urgently needed.

\section{Study Limitations}

This observational and retrospective registry-based analysis reflects a realistic picture of consecutive health care supply of high-risk patients presenting with ventricular tachyarrhythmias. The rate of patients lost to followup regarding the evaluated end point of all-cause mortality was minimal. To minimize this lost to follow-up rate, all patients not meeting ICD follow-up at least once after discharge were excluded from our analysis. Pharmacological therapies were based on discharge medication at the index event. All clinical data was documented reliably by individual cardiologists during routine clinical care who were blinded to the final analyses, alleviating the use of an independent clinical event committee. Our results need to be re-evaluated with the use of larger cohorts and more representative multi-centre registry data, or even RCT, that focus specifically on the effect of LVEF $<35 \%$ in selected subgroups also with additional ablation and ICD therapy.

\section{Statement of Ethics}

The study was carried out according to the principles of the Declaration of Helsinki and was approved by the Medical Ethics Committee II of the Medical Faculty Mannheim, University of Heidelberg, Germany.

\section{Disclosure Statement}

There were no conflicts of interest.

\section{Funding Source}

There was no funding.

\section{References}

1 Priori SG, Blomström-Lundqvist C, Mazzanti A, Blom N, Borggrefe M, Camm J, et al.; ESC Scientific Document Group. 2015 ESC Guidelines for the management of patients with ventricular arrhythmias and the prevention of sudden cardiac death: the Task Force for the Management of Patients with Ventricular Arrhythmias and the Prevention of Sudden Cardiac Death of the European Society of Cardiology (ESC). Endorsed by: Association for European Paediatric and Congenital Cardiology (AEPC). Eur Heart J. 2015 Nov;36(41): 2793-867.

2 Cleland JG, Massie BM, Packer M. Sudden death in heart failure: vascular or electrical? Eur J Heart Fail. 1999 Mar; 1(1):41-5.

3 Gradman A, Deedwania P, Cody R, Massie B, Packer M, Pitt B, et al.; Captopril-Digoxin Study Group. Predictors of total mortality and sudden death in mild to moderate heart failure. J Am Coll Cardiol. 1989 Sep;14(3): $564-70$.

4 Al-Khatib SM, Stevenson WG, Ackerman MJ, et al. AHA/ACC/HRS Guideline for Management of Patients with Ventricular Arrhythmias and the Prevention of Sudden Cardiac Death: a Report of the American College of Cardiology/American Heart Association Task Force on Clinical Practice Guidelines and the Heart Rhythm Society. Circulation. 2018;138:e272-e391.
5 Monserrat L, Elliott PM, Gimeno JR, Sharma S, Penas-Lado M, McKenna WJ. Non-sustained ventricular tachycardia in hypertrophic cardiomyopathy: an independent marker of sudden death risk in young patients. J Am Coll Cardiol. 2003 Sep;42(5):873-9.

6 Adabag AS, Casey SA, Kuskowski MA, Zenovich AG, Maron BJ. Spectrum and prognostic significance of arrhythmias on ambulatory Holter electrocardiogram in hypertrophic cardiomyopathy. J Am Coll Cardiol. 2005 Mar;45(5):697-704.

7 Nava A, Bauce B, Basso C, Muriago M, Rampazzo A, Villanova $C$, et al. Clinical profile and long-term follow-up of 37 families with arrhythmogenic right ventricular cardiomyopathy. J Am Coll Cardiol. 2000 Dec;36(7): 2226-33.

8 Hamid MS, Norman M, Quraishi A, Firoozi S, Thaman R, Gimeno JR, et al. Prospective evaluation of relatives for familial arrhythmogenic right ventricular cardiomyopathy/dysplasia reveals a need to broaden diagnostic criteria. J Am Coll Cardiol. 2002 Oct;40(8): 1445-50.

9 Kjekshus J. Arrhythmias and mortality in congestive heart failure. Am J Cardiol. 1990 May;65(19):42I-8I.

10 Connolly SJ, Hallstrom AP, Cappato R, Schron EB, Kuck KH, Zipes DP, et al. Metaanalysis of the implantable cardioverter defi- brillator secondary prevention trials. AVID CASH and CIDS studies. Antiarrhythmics vs Implantable Defibrillator study. Cardiac Arrest Study Hamburg. Canadian Implantable Defibrillator Study. Eur Heart J. 2000 Dec; 21(24):2071-8

11 Kuck KH, Cappato R, Siebels J, Rüppel R. Randomized comparison of antiarrhythmic drug therapy with implantable defibrillators in patients resuscitated from cardiac arrest: the Cardiac Arrest Study Hamburg (CASH). Circulation. 2000 Aug;102(7):748-54.

12 Antiarrhythmics versus Implantable Defibrillators (AVID) Investigators. A comparison of antiarrhythmic-drug therapy with implantable defibrillators in patients resuscitated from near-fatal ventricular arrhythmias. N Engl J Med. 1997 Nov;337(22):1576-83.

13 Connolly SJ, Gent M, Roberts RS, Dorian P, Roy D, Sheldon RS, et al. Canadian implantable defibrillator study (CIDS): a randomized trial of the implantable cardioverter defibrillator against amiodarone. Circulation. 2000 Mar;101(11):1297-302.

14 Moss AJ, Hall WJ, Cannom DS, Daubert JP, Higgins SL, Klein H, et al.; Multicenter Automatic Defibrillator Implantation Trial Investigators. Improved survival with an implanted defibrillator in patients with coronary disease at high risk for ventricular arrhythmia. $\mathrm{N}$ Engl J Med. 1996 Dec;335(26):1933-40. 
15 Kadish A, Dyer A, Daubert JP, Quigg R, Estes NA, Anderson KP, et al.; Defibrillators in Non-Ischemic Cardiomyopathy Treatment Evaluation (DEFINITE) Investigators. Prophylactic defibrillator implantation in patients with nonischemic dilated cardiomyopathy. N Engl J Med. 2004 May;350(21):21518.

16 Moss AJ, Zareba W, Hall WJ, Klein H, Wilber DJ, Cannom DS, et al.; Multicenter Automatic Defibrillator Implantation Trial II Investigators. Prophylactic implantation of a defibrillator in patients with myocardial infarction and reduced ejection fraction. N Engl J Med. 2002 Mar;346(12):877-83.

17 Køber L, Thune JJ, Nielsen JC, Haarbo J, Videbæk L, Korup E, et al.; DANISH Investigators. Defibrillator Implantation in Patients with Nonischemic Systolic Heart Failure. N Engl J Med. 2016 Sep;375(13):1221-30.

18 Hohnloser SH, Kuck KH, Dorian P, Roberts RS, Hampton JR, Hatala R, et al.; DINAMIT Investigators. Prophylactic use of an implantable cardioverter-defibrillator after acute myocardial infarction. N Engl J Med. 2004 Dec;351(24):2481-8.

19 Bänsch D, Antz M, Boczor S, Volkmer M, Tebbenjohanns J, Seidl K, et al. Primary prevention of sudden cardiac death in idiopathic dilated cardiomyopathy: the Cardiomyopathy Trial (CAT). Circulation. 2002 Mar; 105(12):1453-8.

20 Bristow MR, Saxon LA, Boehmer J, Krueger S, Kass DA, De Marco T, et al.; Comparison of Medical Therapy, Pacing, and Defibrillation in Heart Failure (COMPANION) Investigators. Cardiac-resynchronization therapy with or without an implantable defibrillator in advanced chronic heart failure. $\mathrm{N}$ Engl J Med. 2004 May;350(21):2140-50.

21 Buxton AE, Lee KL, Fisher JD, Josephson ME, Prystowsky EN, Hafley G; Multicenter Unsustained Tachycardia Trial Investigators. A randomized study of the prevention of sudden death in patients with coronary artery disease. N Engl J Med. 1999 Dec;341(25): 1882-90.

22 Zeitler EP, Al-Khatib SM, Friedman DJ, Han JY, Poole JE, Bardy GH, et al. Predicting appropriate shocks in patients with heart failure: patient level meta-analysis from SCD-HeFT and MADIT II. J Cardiovasc Electrophysiol. 2017 Nov;28(11):1345-51.

23 Buxton AE. Should everyone with an ejection fraction less than or equal to $30 \%$ receive an implantable cardioverter-defibrillator? Not everyone with an ejection fraction $[\{L T\}]$ or $=$ $30 \%$ should receive an implantable cardioverter-defibrillator. Circulation. 2005 May; 111(19):2537-49.

24 Wellens HJ, Schwartz PJ, Lindemans FW, Buxton AE, Goldberger JJ, Hohnloser SH, et al. Risk stratification for sudden cardiac death: current status and challenges for the future. Eur Heart J. 2014 Jul;35(25):1642-51.

25 Deneke T, Israel CW, Krug J, Nentwich K, Müller P, Mügge A, et al. Indikationen zur Katheterablation bei ventrikulärer Tachykardie. Dtsch Med Wochenschr. 2013 Sep; 138(39):1952-6.

26 Authors/Task Force M, Dickstein K, Vardas PE, et al. 2010 focused update of ESC guidelines on device therapy in heart failure: an update of the 2008 ESC Guidelines for the diagnosis and treatment of acute and chronic heart failure and the 2007 ESC Guidelines for cardiac and resynchronization therapy. Developed with the special contribution of the Heart Failure Association and the European Heart Rhythm Association. Europace. 2010; 12(11):1526-36.

27 Ponikowski P, Voors AA, Anker SD, Bueno $\mathrm{H}$, Cleland JG, Coats AJ, et al.; Authors/Task Force Members; Document Reviewers. 2016 ESC Guidelines for the diagnosis and treatment of acute and chronic heart failure: the Task Force for the diagnosis and treatment of acute and chronic heart failure of the European Society of Cardiology (ESC). Developed with the special contribution of the Heart
Failure Association (HFA) of the ESC. Eur J Heart Fail. 2016 Aug;18(8):891-975.

28 Austin PC. An Introduction to Propensity Score Methods for Reducing the Effects of Confounding in Observational Studies. Multivariate Behav Res. 2011 May;46(3):399-424.

29 Ferdinand D, Otto M, Weiss C. Get the most from your data: a propensity score model comparison on real-life data. Int J Gen Med. 2016 May;9:123-31.

30 Schaer B, Theuns DA, Sticherling C, Szili-Torok T, Osswald S, Jordaens L. Effect of implantable cardioverter-defibrillator on left ventricular ejection fraction in patients with idiopathic dilated cardiomyopathy. Am J Cardiol. 2010 Dec;106(11):1640-5.

31 Zhang Y, Guallar E, Blasco-Colmenares E, Butcher B, Norgard S, Nauffal V, et al. Changes in Follow-Up Left Ventricular Ejection Fraction Associated with Outcomes in Primary Prevention Implantable Cardioverter-Defibrillator and Cardiac Resynchronization Therapy Device Recipients. J Am Coll Cardiol. 2015 Aug;66(5):524-31.

32 Masarone D, Limongelli G, Rubino M, Valente F, Vastarella R, Ammendola E, et al. Management of Arrhythmias in Heart Failure. J Cardiovasc Dev Dis. 2017 Feb;4(1):E3.

33 Seegers J, Bergau L, Expósito PM, Bauer A, Fischer TH, Lüthje L, et al. Prediction of Appropriate Shocks Using 24-Hour Holter Variables and T-Wave Alternans after First Implantable Cardioverter-Defibrillator Implantation in Patients with Ischemic or Nonischemic Cardiomyopathy. Am J Cardiol. 2016 Jul;118(1):86-94.

34 Schliamser JE, Kadish AH, Subacius H, Shalaby A, Schaechter A, Levine J, et al.; DEFINITE Investigators. Significance of follow-up left ventricular ejection fraction measurements in the Defibrillators in Non-Ischemic Cardiomyopathy Treatment Evaluation trial (DEFINITE). Heart Rhythm. 2013 Jun;10(6): $838-46$. 